\title{
Computational comparison of different plating strategies in medial open-wedge high tibial osteotomy with lateral hinge fractures
}

Yen-Nien Chen ${ }^{1 * \dagger} \mathbb{B}$, Chang-Han Chuang ${ }^{2 \dagger}$, Tai-Hua Yang ${ }^{3,4,5,6,7}$, Chih-Wei Chang ${ }^{4,7^{*}}$, Chun-Ting Li ${ }^{8}$, Chia-Jung Chang ${ }^{3}$ and Chih-Han Chang ${ }^{3,6}$

\begin{abstract}
Background: Lateral hinge fracture (LHF) is associated with nonunion and plate breakage in high tibial osteotomy (HTO). Mechanical studies investigating fixation strategies for LHFs to restore stability and avoid plate breakage are absent. This study used computer simulation to compare mechanical stabilities in HTO for different LHFs fixed with medial and bilateral locking plates.

Methods: A finite element knee model was created with HTO and three types of LHF, namely T1, T2, and T3 fractures, based on the Takeuchi classification. Either medial plating or bilateral plating was used to fix the HTO with LHFs. Furthermore, the significance of the locking screw at the combi hole (D-hole) of the medial TomoFix plate was evaluated.

Results: The osteotomy gap shortening distance increased from 0.53 to $0.76,0.79$, and $0.72 \mathrm{~mm}$ after T1, T2, and T3 LHFs, respectively, with medial plating only. Bilateral plating could efficiently restore stability and maintain the osteotomy gap. Furthermore, using the D-hole screw reduced the peak stress on the medial plate by $28.7 \%$ (from 495 to $353 \mathrm{MPa}$ ), 26.6\% (from 470 to $345 \mathrm{MPa}$ ), and 32.6\% (from 454 to $306 \mathrm{MPa}$ ) in T1, T2, and T3 LHFs, respectively.

Conclusion: Bilateral plating is a recommended strategy to restore HTO stability in LHFs. Furthermore, using a Dhole locking screw is strongly recommended to reduce the stress on the medial plate for lowering plate breakage risk.
\end{abstract}

Keywords: High tibial osteotomy, Lateral hinge fracture, Plating strategy, Finite element simulation

\footnotetext{
*Correspondence: yennien.chen@gmail.com; 108100040@gm.asia.edu.tw; u7901064@yahoo.com.tw

†Yen-Nien Chen and Chang-Han Chuang contributed equally to this work.

'Department of Physical Therapy, Asia University, 500, Lioufeng Rd., Wufeng, Taichung 41354, Taiwan

${ }^{4}$ Department of Orthopedics, National Cheng Kung University Hospital, College of Medicine, National Cheng Kung University, Tainan, Taiwan

Full list of author information is available at the end of the article
}

(c) The Author(s). 2020 Open Access This article is licensed under a Creative Commons Attribution 4.0 International License, which permits use, sharing, adaptation, distribution and reproduction in any medium or format, as long as you give appropriate credit to the original author(s) and the source, provide a link to the Creative Commons licence, and indicate if changes were made. The images or other third party material in this article are included in the article's Creative Commons licence, unless indicated otherwise in a credit line to the material. If material is not included in the article's Creative Commons licence and your intended use is not permitted by statutory regulation or exceeds the permitted use, you will need to obtain permission directly from the copyright holder. To view a copy of this licence, visit http://creativecommons.org/licenses/by/4.0/. The Creative Commons Public Domain Dedication waiver (http://creativecommons.org/publicdomain/zero/1.0/) applies to the data made available in this article, unless otherwise stated in a credit line to the data. 


\section{Introduction}

High tibial osteotomy (HTO) with a locking plate is a well-established surgical approach to adjust the mechanical axis of the low extremity with medial compartmental knee osteoarthritis (KOA) and restore the joint space of the medial knee of the KOA [1-3]. In recent years, HTO has gained popularity because of its satisfactory clinical outcome, particularly in patients aged 40-60 years [4-6]. Furthermore, HTO slows down the KOA process, and approximately $90 \%$ of patients could resume their original work and sports within 1 year of HTO [7]. To ensure the bone healing of the medial open wedge after HTO, stable fixation is required, and recently, the locking plate has been shown to provide excellent stability for early rehabilitation exercise $[8,9]$.

Although HTO showed satisfactory clinical outcomes, complications such as the need for symptomatic hardware removal, deep wound infection, hardware failure with correction loss, nonunion, early conversion to arthroplasty, displaced $(>2 \mathrm{~mm})$ and undisplaced $(<2 \mathrm{~mm})$ lateral hinge fracture (LHF), delayed wound healing, and undisplaced lateral tibia plateau fracture are common [10]. Other studies have reported an LHF incidence of 19.841.2\% after HTO [7, 11-13]. Furthermore, LHF is highly related to nonunion and plate breakage [14].

Many studies have investigated LHF incidence after HTO and complications following LHFs. The biomechanical effect of the LHFs on the HTO with medial opening wedge and the bone plate was also revealed [15]. In the study, the stress of the medial plate was increased with the LHFs. However, no mechanical study has investigated the fixation strategies of HTO for LHFs to restore stability and avoid plate breakage. Bilateral plating was demonstrated to re-correct the loss and nonunion after HTO [16], whereas mechanical stability was not investigated. Furthermore, only one type of LHF was involved in the study [16]; the other types of LHFs were not investigated.

The aim of this study was to compare the mechanical stability, including gap shortening at the osteotomy site, the displacement of the proximal tibial fragment, and the stress on the metallic bone plates, in HTO with various LHFs, and medial and bilateral plates by using the finite element (FE) method. The FE method is a numerical tool to obtain mechanical responses, including internal stress and deformation, of the whole model [17, 18]. Additionally, the FE method excludes the variation between the samples in the study.

\section{Methods}

A reliable FE HTO model, which was validated and used to compare HTO stability with various screw configurations in a previous study [19], was modified to conduct HTO with LHFs and following medial and bilateral plate fixation.

\section{Solid model}

The HTO model, with medial TomoFix plate and without LHF, used in a previous study was modified according to various hinge fractures. The angle of the medial open wedge and the width of the lateral hinge were set to $12^{\circ}$ and $10 \mathrm{~mm}$, respectively. The width of the lateral hinge refers to the distance from the apex of the wedge to the lateral edge of the tibia. In this study, three types of LHF fractures, namely type 1 (T1), type 2 (T2), and type 3 (T3), were created according to the Takeuchi classification [20]. $\mathrm{T} 1$ fracture referred to a crack, parallel to the osteotomy line of the open wedge, in the open wedge extending to the proximal site of the tibiofibular joint. T2 fracture referred to a crack in the open wedge extending downwardly to the distal site of the tibiofibular joint. T3 fracture was a lateral plateau fracture, which referred to a crack upwardly to the lateral plateau of the proximal tibia (Fig. 1). The fractures were created with virtual planes using the CAD software SOLIDWORKS 2019 (Dassault Systemes SolidWorks Corp., Waltham, MA, USA). Hence, the fracture surfaces were simplified as planes, and no gap existed at the fracture sites. The micro-geometry of the fractured surface was not considered. Then, different fixation strategies were used to stabilize each fracture type (Fig. 2), including medial plating (MP) with the original TomoFix plate (TomoFix, DePuy Synthes, Oberdorf, Switzerland), bilateral plating (BP) with medial TomoFix plate and proximal lateral tibia locking plate (LCP, proximal tibia plate, DePuy Synthes, Oberdorf, Switzerland), and BP without the locking screw (BPWDS) at the proximal combi hole (D-Hole) of the TomoFix plate. The length and width of the TomoFix plate were 115 and $16 \mathrm{~mm}$, respectively. The thickness of both medial TomoFix and lateral locking plates was set to $3 \mathrm{~mm}$. The lengths of the screw at the proximal tibia (including the D-hole screw) and tibia shift in the MP were 56 and $26 \mathrm{~mm}$, respectively. However, the length of the D-hole screw in the BP group was reduced to $38 \mathrm{~mm}$ to dodge the proximal screw of the lateral locking plate. The length of the proximal screw for the lateral locking plate was $56 \mathrm{~mm}$ (top row) and $44 \mathrm{~mm}$ (second and third row from the top) (Fig. 2b, c). The screw length for the lateral locking plate at the tibia shift was $26 \mathrm{~mm}$. The diameter of all screws was set to $5 \mathrm{~mm}$.

\section{FE model}

The models were imported into the ANSYS Workbench 2019 R3 (Swanson Analysis Systems, Inc., Houston, PA, USA) for the following mesh and simulation. Quadratic tetrahedral element (solid 187) was used to mesh hole the model with commend "free mesh." The local element sizes, including screws, plates, cartilage, and the surfaces of the bone in contact with the screws, were refined with commend "sizing." Totally, 737,119 elements and 1,173,791 nodes were used to mesh the MP model. The same settings 


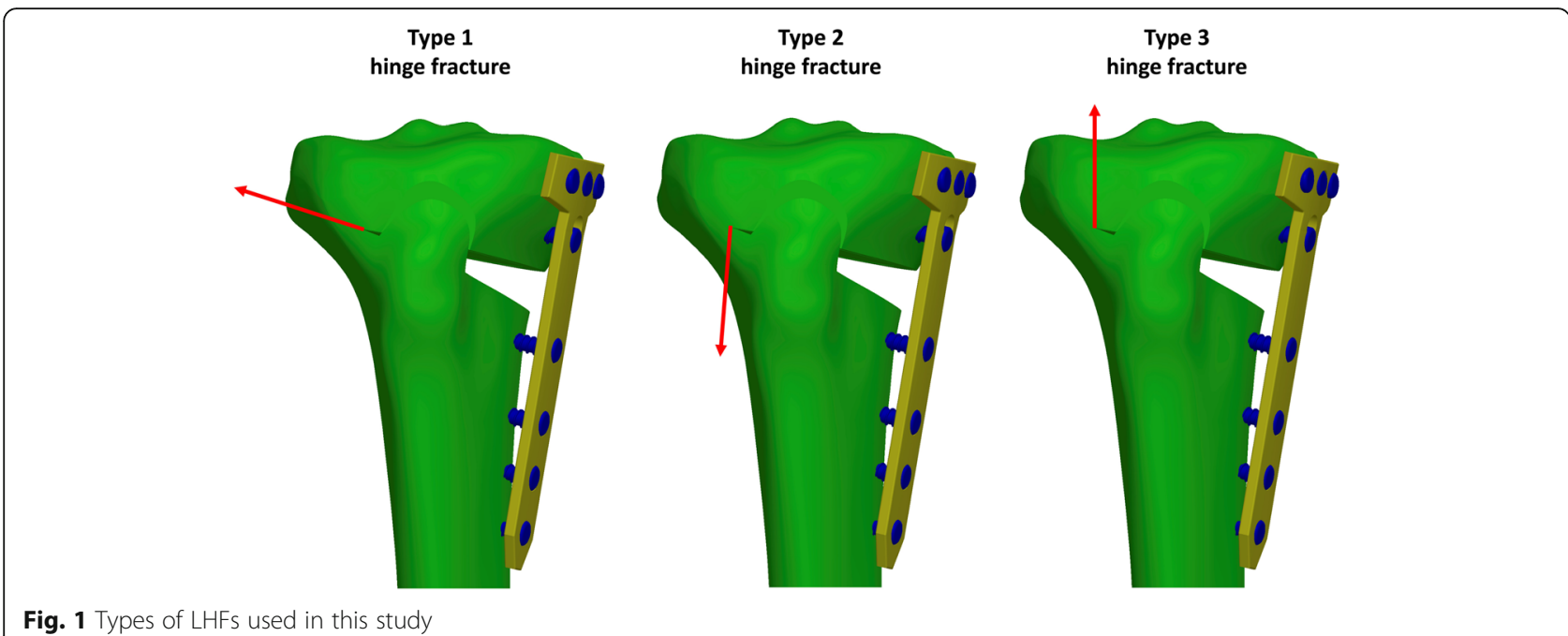

were used to mesh the other two models. Four ligaments (Table 1), namely medial collateral ligament, lateral collateral ligament, anterior cruciate ligament, and posterior cruciate ligament (Fig. 2d), around the knee joint were reconstructed with tension-only spring in the ANSYS Workbench. The locations of the springs were defined based on the anatomy. The contact behavior between the screw and plates was set to bond to simulate the locking mechanism of the locking plate and locking screw. The contact behavior between the screws and the surrounding bone was set to frictional surface-to-surface contact behavior. Furthermore, the bone-to-bone contact behavior at the fracture site and the osteotomy site was set to the frictional surface-to-surface contact. The frictional coefficients of the bone-to-bone and bone-to-metal were defined as 0.45 and 0.3 , respectively, based on the literature [21]. The contact behavior between the bone and meniscus of the knee joint was set to frictionless.

\section{Material properties and boundary condition}

The material property of the locking plate ad screws was set to titanium, and the elastic modulus and Poisson's ratio were defined according to the engineering database in the ANSYS Workbench. The material properties of the cortical bone, cancellous bone, and meniscus were defined based on the literature [22-24] (Table 2). A $2000-\mathrm{N}$ vertical downward force (approximately 2.5 times the body weight of an $80-\mathrm{kg}$ subject) was applied on the upper surface of the distal femur to simulate the maximum force on the knee while walking [25]. Only the motion in the vertical direction was allowed for the

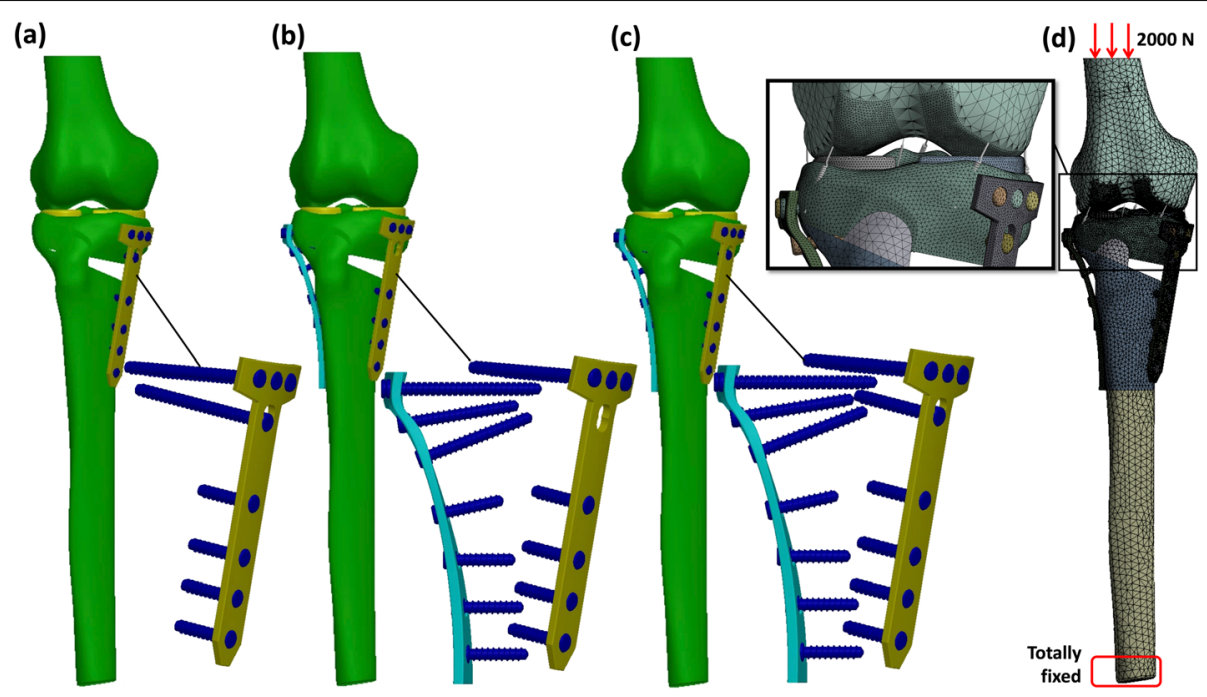

Fig. 2 Fixation strategies and bonudary condition used in this study, including a MP, b BP without D-hole screw, c BP, and d boundary condition 
Table 1 Material properties of the springs used in this study to simulate ligaments

\begin{tabular}{lllll}
\hline Ligament & $\begin{array}{l}\text { Numbers } \\
\text { of spring }\end{array}$ & $\begin{array}{l}\text { Stiffness } \\
(\mathrm{N} / \mathrm{mm})\end{array}$ & $\begin{array}{l}\text { Cross-section } \\
\text { area }\left(\mathrm{mm}^{2}\right)\end{array}$ & $\begin{array}{l}\text { Elastic } \\
\text { modulus } \\
(\mathrm{MPa})\end{array}$ \\
\hline Medial collateral ligament & 2 & 10.6 & 1.54 & 345 \\
Lateral collateral ligament & 2 & 10.6 & 1.54 & 345 \\
$\begin{array}{l}\text { Anterior cruciate ligament } \\
\text { Posterior cruciate }\end{array}$ & 1 & 17.5 & 1.29 & 345 \\
ligament & & 20.6 & 1.92 & 345 \\
\hline
\end{tabular}

distal femur. The distal surface of the tibia was totally fixed (Fig. 2d).

\section{Criteria}

The maximum displacement of the proximal osteotomy fragment and shortening of the osteotomy gap were adopted as indexes to compare HTO stability with LHFs and fixation approaches. The equivalent stress (also called von Mises stress) on plates was used as an index to evaluate the breakage risk. Furthermore, the force on nodes at the transverse plane of the medial plate below the D-hole was calculated to compare the loading on the medial plate with and without LHFs.

\section{Result}

\section{Stability}

Results indicated that LHFs substantially reduced HTO stability when only the medial plate was used, whereas the stability was restored with the addition of the lateral plate, irrespective of the screw used at the D-hole locking (Figs. 3, 4, and 5). The maximum displacement of the proximal tibia fragment with only the MP increased by $42 \%$ (from 0.88 to $1.25 \mathrm{~mm}$ ), $48.9 \%$ (from 0.88 to $1.31 \mathrm{~mm}$ ), and $34.1 \%$ (from 0.88 to $1.18 \mathrm{~mm}$ ) after $\mathrm{T} 1$, T2, and T3 LHFs, respectively (Fig. 6a). Additionally, the osteotomy gap shortening distance increased by $43.4 \%$ (from 0.53 to $0.76 \mathrm{~mm}$ ), $49 \%$ (from 0.53 to $0.79 \mathrm{~mm}$ ), and $35.8 \%$ (from 0.53 to $0.72 \mathrm{~mm}$ ), respectively, after $\mathrm{T} 1$, T2, and T3 LHFs (Fig. 6). The BP could restore the stability through the reduction in fragment displacement by $32 \%$ (from 1.25 to $0.85 \mathrm{~mm}$ ), $29.8 \%$ (from 1.31 to 0.92 $\mathrm{mm}$ ), and $55.2 \%$ (from 1.81 to $0.81 \mathrm{~mm}$ ) in T1, T2, and T3 LHFs, respectively (Fig. 6a). Furthermore, the BP could maintain the osteotomy gap. The gap shortening distance in HTO without LHFs was $0.53 \mathrm{~mm}$; after LHFs

Table 2 Material properties used in this study

\begin{tabular}{lll}
\hline & Elastic modulus (MPa) & Poisson ratio \\
\hline Cortical bone & 12000 & 0.3 \\
Cancellous bone & 430 & 0.3 \\
Meniscus & 100 & 0.1 \\
Titanium alloy & 96000 & 0.36 \\
\hline
\end{tabular}

with $\mathrm{BP}$, the gap shorting distances were 0.51, 0.54, and $0.48 \mathrm{~mm}$ in T1, T2, and T3 LHFs, respectively (Fig. 6b). Moreover, using the locking screw at the D-hole (BP) reduced the fragment displacement compared with that without the screw (BPWDS).

\section{Loading on the medial plate}

Loading on the medial plate increased slightly after T1 and T3 LHFs but decreased slightly after T2 LHF when only the medial plate was used. However, the loading on the medial plate decreased after adding the lateral plate in LHFs. Using the D-hole screw at the medial plate did not affect loading on the medial plate. The loading on the medial plate in HTO was $820 \mathrm{~N}$, which increased by $51 \mathrm{~N}$ and $23 \mathrm{~N}$ but decreased by $24 \mathrm{~N}$ after T1, T3, and T2 LHFs, respectively (Fig. 6c).

\section{Stress on the plates}

The peak equivalent stress on the medial plate clearly increased after LHF. The peak stress on the medial plate increased by $20.8 \%$ (from 331 to $400 \mathrm{MPa}$ ), $23.9 \%$ (from 331 to $410 \mathrm{MPa}$ ), and $16.3 \%$ (from 331 to $385 \mathrm{MPa}$ ) (Fig. 7). Using the lateral plate plus the D-hole screw (BP group) could efficiently reduce the peak stress on the medial plate after LHFs. Moreover, the peak stress on the medial plate increased after LHFs without the D-hole screw although the lateral plate was added. However, using the D-hole screw did not alter the stress on the lateral plate (Fig. 8).

\section{Discussion}

Using HTO for medial compartmental KOA is currently popular because the locking plate and screws can provide excellent stability immediately after osteotomy, enabling the patient to start rehabilitation programs earlier than with the use of the traditional compression plate. Scholars have shown that MP with only the TomoFix locking plate stabilizes HTO with a medial open wedge (without LHF) and provides enough stability for bone healing [26-28]. However, when the lateral hinge is fractured, MP only does not provide adequate stability for bone healing at the osteotomy site and at the fractured lateral hinge. Furthermore, LHFs increased the stress on the medial plate and plate breakage risk. Stability decreases while plate stress increases because the continuous bony structure of the lateral hinge is broken. In this situation, the original medial plate (which is at a distance from the fractured lateral hinge) only was insufficient to stabilize the osteotomized and fractured HTO. Hence, additional fixation, such as lateral plating, is needed for HTO after LHFs.

Mechanically, the medial open wedge is the weakest site of the HTO structure, irrespective of the presence of LHFs. Because no bone medially supports the proximal 

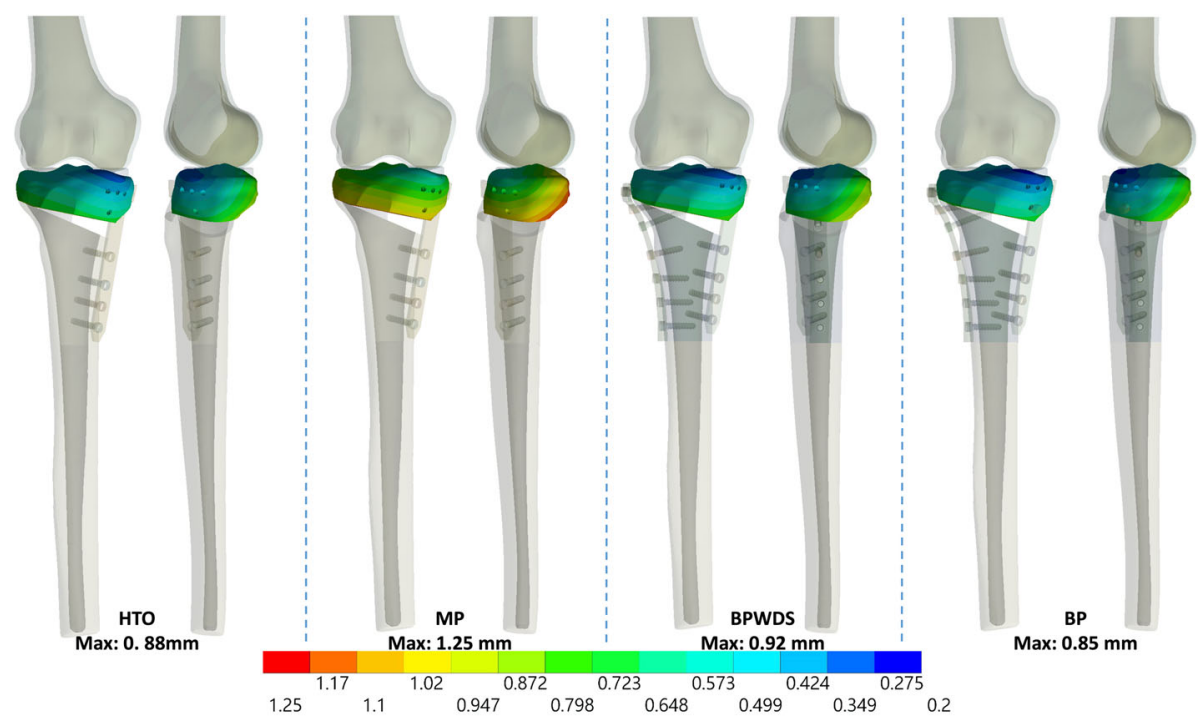

Fig. 3 Total displacement of the proximal tibia fragment in HTO and T1 LHF

tibial fragment, a strong support, such as the locking plate, is necessary to substitute the medial bone function. Although the fractured bone at the lateral hinge was able to sustain partial loading, the compressive force, the fractured bone was unable to resist the developed shear or tensile force during the compressive loading in the present simulation. Furthermore, LHFs were at a distance from the MP, and the fixation effect of the medial plate was minimal for LHFs. In such a situation, placing a lateral plate directly at the fractured lateral hinge could efficiently stabilize the tibia with HTO and LHFs.

Plate breakage near the D-hole was highly related to LHFs in a previous study [14], and the result was in accordance with the present FE results. A relative highstress area appeared near the outside corner and the Dhole of the medial TomoFix plate in HTO, particularly with LHFs in the present study. In the previous FE study [15], the stress of the plate was also increased, particularly in the T2 and T3 LHFs. The high stress after LHFs can create a crack and then extend to the screw hole after cyclic loading during postsurgical walking without bone healing. In the present simulation, BP with the Dhole screw proved to reduce the stress on the medial plate; hence, this approach is recommended in HTO for LHFs.

The locking screw at the D-hole of the medial TomoFix plate is crucial for reducing stress on the plate in BP.

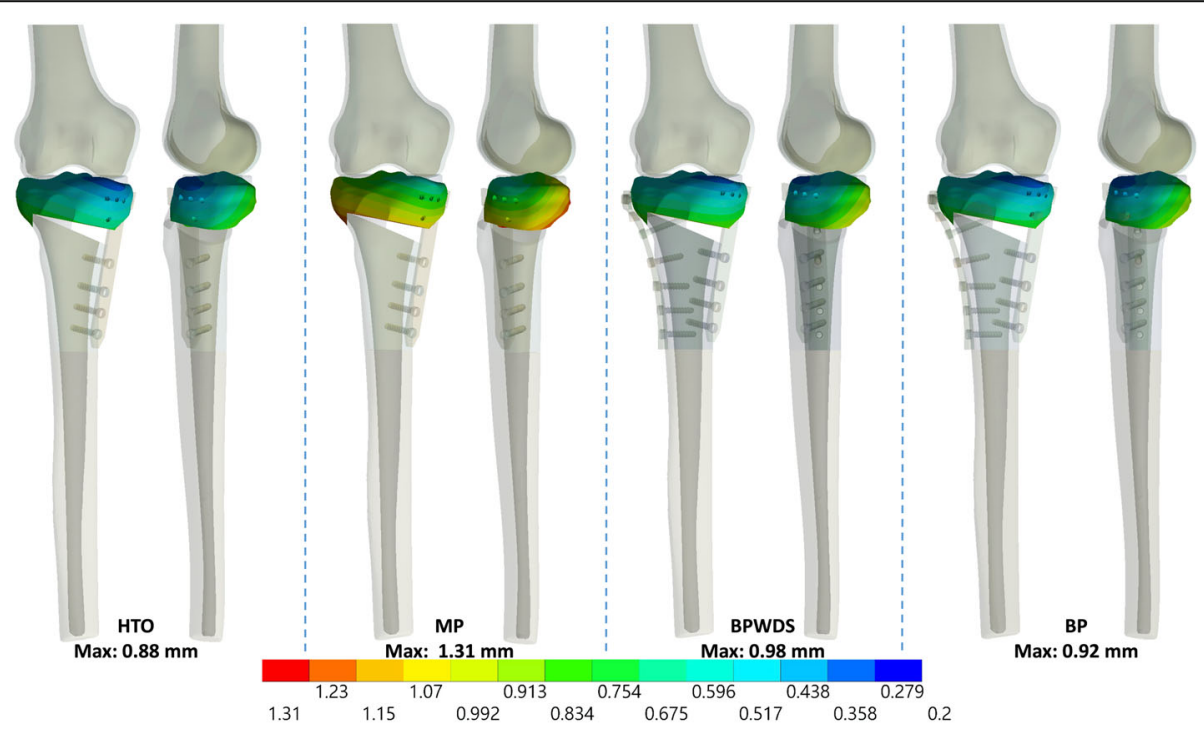

Fig. 4 Total displacement of the proximal tibia fragment in HTO and T2 LHF 


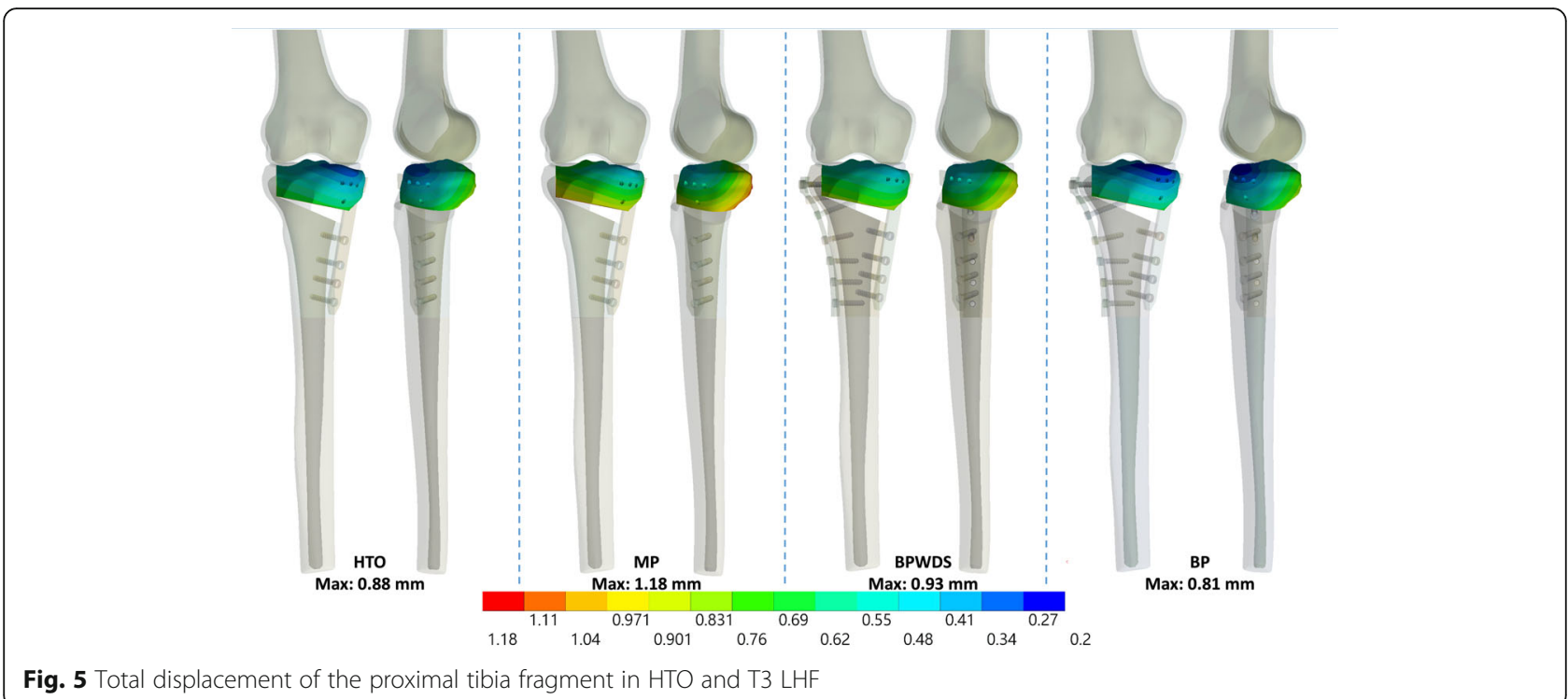

In the present simulation, although the stability of the BPWDS was close to that in the BP group, the equivalent stress on the medial plate in BPWDS was much higher than that in the BP group. Furthermore, the equivalent stress on the plate in the BPWDS group was even higher than that in the MP group. The results of the stress indicated the contribution of the D-hole screw to reduce the plate stress. Although the difference in the total loading of the medial plate with and without the screw at the D-hole in bilateral plating was minor, the difference in stress was large. This is because the D-hole screw shunts the force and alerts the total moment developed by the applied loading on the medial plate. Finally, the stress on the plate was lower with the D-hole screw than that without the D-hole screw in BP. In clinical practice, the surgeon may encounter challenges while inserting the D-hole screw. However, the D-hole screw is indispensable for HTO in BP, and the surgeon must prioritize inserting the D-hole screw during HTO for LHFs in BP.
The major concern with BP on the HTO for LHFs is the stress-shielding effect, which leads to a stiff structure during bone healing, leading to callus formation. Recently, the far cortical locking screw and plate have been proposed to reduce structural stiffness and to increase the micromotion of the fracture gap in HTO [29]. In our previous study, the far cortical locking screw reduced the stiffness through an increase in the motion between fragments while increasing the stress on the implant in HTO. Hence, using a far cortical locking screw could be considered a potential strategy for reducing the structural stiffness of the HTO for LHFs by using BP.

T3 LHF is regarded as the worst case among LHFs because the fracture affects the articular surface of the knee joint. Although in the present study the mechanical stability in $\mathrm{T} 3$ was better than that in $\mathrm{T} 1$ and $\mathrm{T} 2$, this does not indicate that T3 LHF is less serious than T1 and T2 LHFs because the deformation of the knee joint surface was not considered in the present simulation. T3

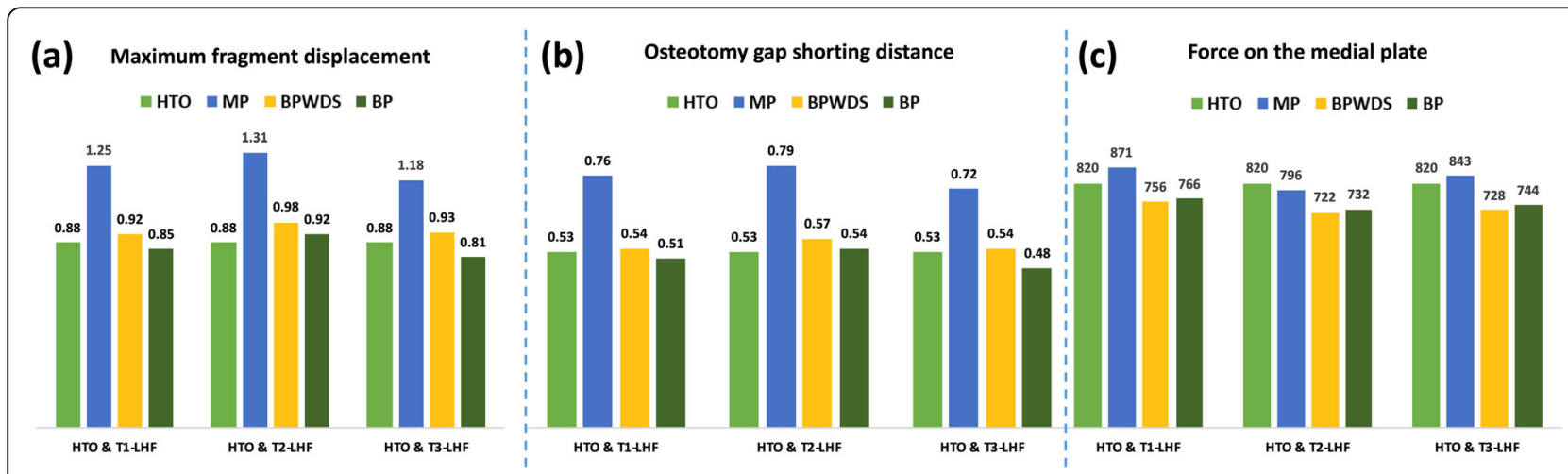

Fig. 6 a Maximum displacement of the proximal tibia fragment. b Gap shortening at the osteotomy site. c Total force on the medial plate 


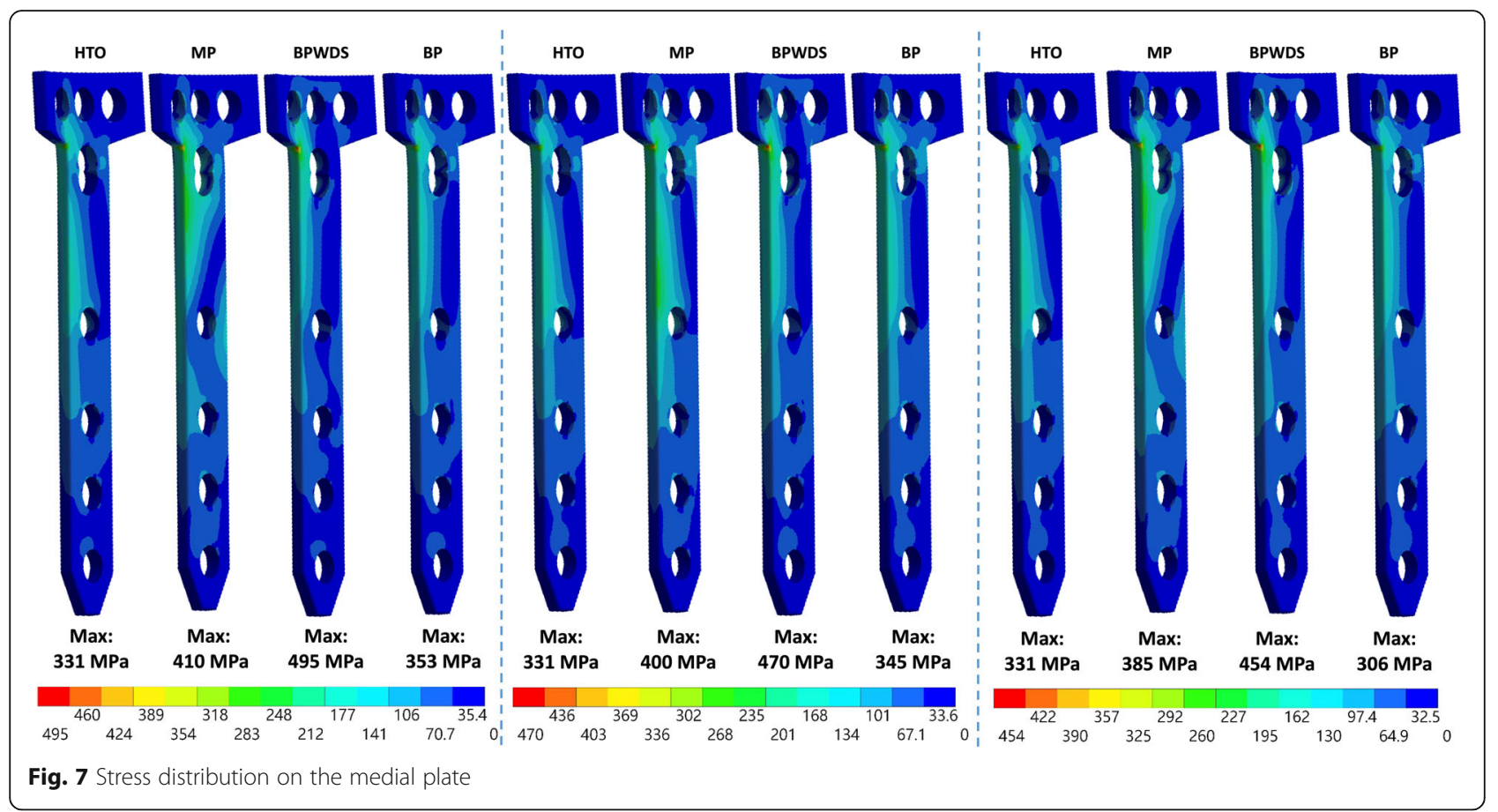

LHF should be treated carefully because the consistency of the articular surface is crucial in the management of an intra-articular fracture. The result of the present mechanical stability is not equal to the final result of bone healing and the functional outcome.

This study has some limitations. First, only the maximum compressive loading conditions in walking were considered. The effect of fixation approaches in the swine phase and tensile loading was not considered. Second, the fibula was not simulated, the trabecular structure of the bone and the micro-geometry of the fractured bone were not considered, and the material properties were simplified as linear elastic, isotropic, and homogeneous. Third, the ligaments around the knee

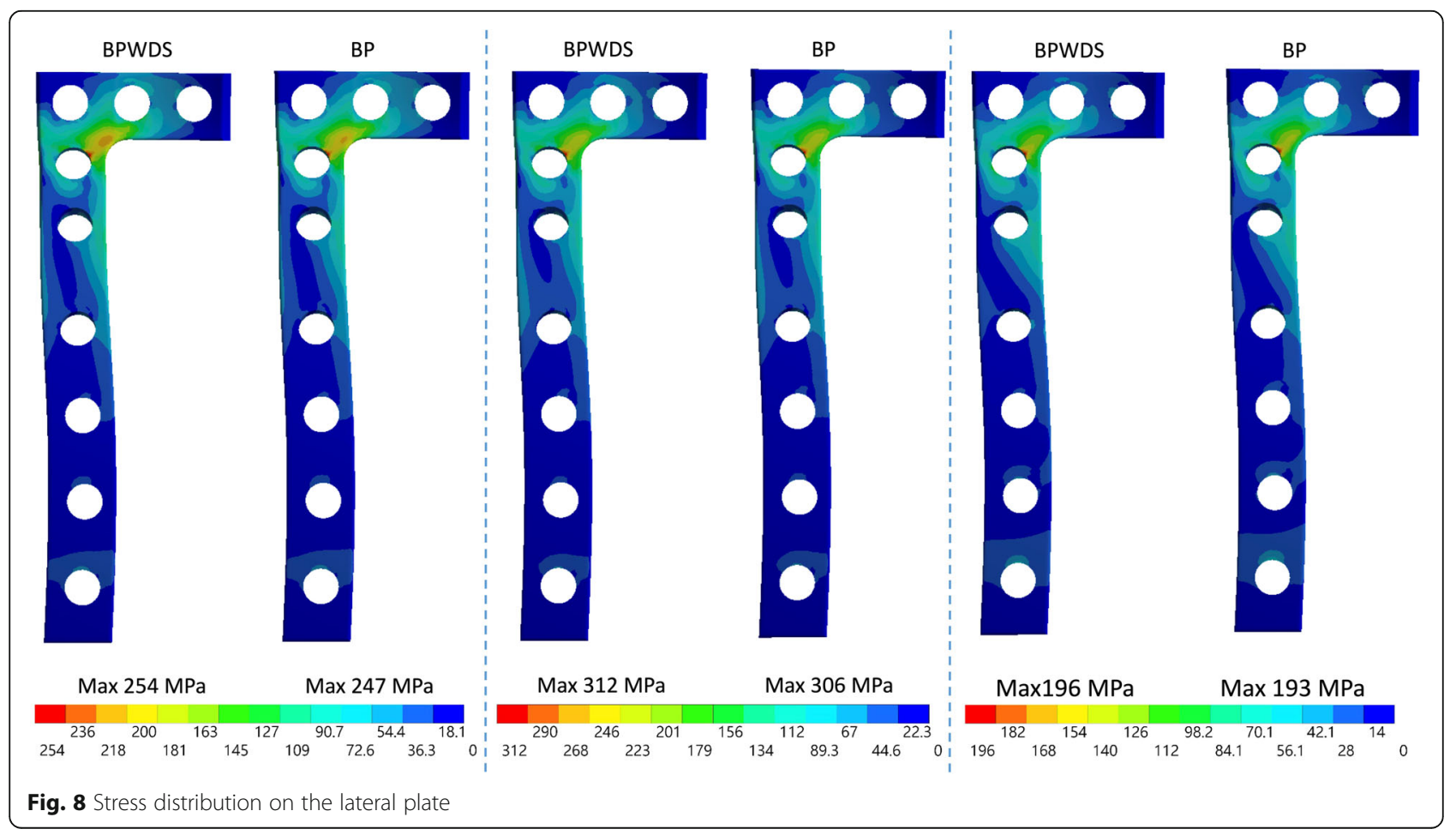


joint were simplified as one-dimensional tension-only springs. Fourth, the original bony structure used was an intact knee without $\mathrm{KOA}$, and only the bone volume of the medial open wedge was removed. The stress developed during the distraction of the open wedge was not considered. Finally, the residual stress between the bone and plate developed during the screw inserting was not considered.

\section{Conclusion}

LHFs drastically reduce HTO stability; hence, a fixation, in addition to the original medial TomoFix plate, is required to restore HTO stability in LHFs. Therefore, bilateral plating is a recommended fixation strategy to restore HTO stability in LHFs. Furthermore, the locking screw at the proximal combi hole (D-hole) is strongly recommended to reduce the stress on the TomoFix plate, thus reducing the risk of plate breakage in the future during postsurgery rehabilitation programs.

\section{Abbreviations}

BP: Bilateral plating; BPWD: Bilateral plating without the locking screw at the proximal combi hole; D-Hole: Combi hole; FE: Finite element; HTO: High tibial osteotomy; KOA: Knee osteoarthritis; LHF: Lateral hinge fracture; MP: Medial plating; T1: Type 1; T2: Type 2; T3: Type 3

\section{Acknowledgements}

Yen-Nien Chen and Chang-Han Chuang contribute equally to this study. The authors also acknowledge the US National Library of Medicine (NLM) and the Visible Human Project for the image data used to create the numerical model in this study.

\section{Authors' contributions}

Conceptualization: Yen-Nien Chen. Data curation: Chang-Han Chuang. Methodology: Tai-Hua Yang. Supervision: Chih-Han Chang. Validation: Chia-Jung Chang. Writing - original draft: Chih-Wei Chang. Writing-review and editing: Chun-Ting Li. The author(s) read and approved the final manuscript.

\section{Funding}

This research was supported by the Show-Chwan Memorial Hospital (Taiwan) grant number NCKUSCMH10806.

\section{Availability of data and materials}

All the data will be available upon motivated request to the corresponding authors of the present paper.

\section{Ethics approval and consent to participate}

This article does not contain any studies with human participants or animals performed by any of the authors.

\section{Consent for publication}

Not applicable

\section{Competing interests}

The authors declare no conflict of interest.

\section{Author details}

'Department of Physical Therapy, Asia University, 500, Lioufeng Rd., Wufeng, Taichung 41354, Taiwan. ²Department of Orthopedics, Show Chwan Memorial Hospital, Changhua City, Taiwan. ${ }^{3}$ Department of Biomedical Engineering, National Cheng Kung University, Tainan, Taiwan. ${ }^{4}$ Department of Orthopedics, National Cheng Kung University Hospital, College of Medicine, National Cheng Kung University, Tainan, Taiwan. ${ }^{5}$ Skeleton Materials and Bio-compatibility Core Lab, Research Center of Clinical Medicine, National Cheng Kung University Hospital, College of Medicine,
National Cheng Kung University, Tainan, Taiwan. ${ }^{6}$ Medical Device Innovation Center, National Cheng Kung University, Tainan, Taiwan. 'Department of Orthopedics, College of Medicine, National Cheng Kung University, Tainan, Taiwan. ${ }^{8}$ Institute of Geriatric Welfare Technology \& Science, Mackay Medical College, New Taipei City, Taiwan.

Received: 23 March 2020 Accepted: 24 August 2020

Published online: 14 September 2020

References

1. Staubli AE, De Simoni C, Babst R, Lobenhoffer P. TomoFix: a new LCPconcept for open wedge osteotomy of the medial proximal tibia--early results in 92 cases. Injury. 2003;34(Suppl 2):B55-62.

2. Staubli AE, Jacob HA. Evolution of open-wedge high-tibial osteotomy: experience with a special angular stable device for internal fixation without interposition material. Int Orthop. 2010;34:167-72.

3. Lobenhoffer P, Agneskirchner JD. Improvements in surgical technique of valgus high tibial osteotomy. Knee Surg Sports Traumatol Arthrosc. 2003;11: 132-8.

4. Floerkemeier S, Staubli AE, Schroeter S, Goldhahn S, Lobenhoffer P. Outcome after high tibial open-wedge osteotomy: a retrospective evaluation of 533 patients. Knee Surg Sports Traumatol Arthrosc. 2013;21: 170-80.

5. Losina E, Katz JN. Total knee arthroplasty on the rise in younger patients: are we sure that past performance will guarantee future success? Arthritis Rheum. 2012:64:339-41.

6. Khan M, Evaniew N, Bedi A, Ayeni OR, Bhandari M. Arthroscopic surgery for degenerative tears of the meniscus: a systematic review and meta-analysis. CMAJ. 2014;186:1057-64

7. Ekhtiari S, Haldane CE, de Sa D, Simunovic N, Musahl V, Ayeni OR. Return to work and sport following high tibial osteotomy: a systematic review. J Bone Joint Surg Am. 2016;98:1568-77.

8. $\quad$ Frigg R, et al. Injury. 2001;32(Suppl 2):63-6.

9. Perren SM. Evolution of the internal fixation of long bone fractures. The scientific basis of biological internal fixation: choosing a new balance between stability and biology. J Bone Joint Surg Br. 2002:84:1093-110

10. Han SB, In Y, Oh KJ, Song KY, Yun ST, Jang KM. Complications associated with medial opening-wedge high tibial osteotomy using a locking plate: a multicenter study. J Arthroplast. 2019:34:439-45.

11. Nakamura R, Komatsu N, Fujita K, Kuroda K, Takahashi M, Omi R, et al. Appropriate hinge position for prevention of unstable lateral hinge fracture in open wedge high tibial osteotomy. Bone Joint J. 2017;99-B:1313-8.

12. Dorofeev A, Tylla A, Benco M, Drescher W, Stangl R. Opposite hinge fractures in high tibial osteotomy: a displacement subtype is more critical than a fracture type. Eur J Orthop Surg Traumatol. 2019.

13. Nha KW, Shin MJ, Suh DW, Nam YJ, Kim KS, Kyung BS: Uniplane medial opening wedge high tibial osteotomy relative to a biplane osteotomy can reduce the incidence of lateral-hinge fracture. Knee Surg Sports Traumatol Arthrosc 2019.

14. Nha KW, Jung WH, Koh YG, Shin YS. D-hole breakage of 2 angular stable locking plates for medial opening-wedge high tibial osteotomy: analysis of results from 12 cases. Medicine (Baltimore). 2019:98:e14138.

15. Kang KT, Koh YG, Lee JA, Lee JJ, Kwon SK. Biomechanical effect of a lateral hinge fracture for a medial opening wedge high tibial osteotomy: finite element study. J Orthop Surg Res. 2020;15:63.

16. Nakamura R, Nishimura $H$, Katsuki Y. Re-correction osteotomy with osteophyte graft for correction loss with non-union after high tibial osteotomy. BMJ Case Rep. 2017;2017.

17. Chang CW, Chung YH, Chang CJ, Chen YN, Li CT, Chang CH, et al. Computational comparison of bone cement and poly aryl-ether-etherketone spacer in single-segment posterior lumbar interbody fusion: a pilot study. Australas Phys Eng Sci Med. 2019.

18. Chang CW, Chen YN, Li CT, Chung CR, Chang CH, Peng YT. Finite element study of the effects of fragment shape and screw configuration on the mechanical behavior of tibial tubercle osteotomy. J Orthop Surg (Hong Kong). 2019;27:2309499019861145.

19. Chen YN, Chang CW, Li CT, Chen $\mathrm{CH}$, Chung CR, Chang $\mathrm{CH}$, et al. Biomechanical investigation of the type and configuration of screws used in high tibial osteotomy with titanium locking plate and screw fixation. J Orthop Surg Res. 2019;14:35 
20. Takeuchi R, Ishikawa H, Kumagai K, Yamaguchi Y, Chiba N, Akamatsu Y, et al. Fractures around the lateral cortical hinge after a medial opening-wedge high tibial osteotomy: a new classification of lateral hinge fracture. Arthroscopy. 2012;28:85-94

21. Cordey J, Mikuschka-Galgoczy E, Blumlein H, Schneider U, Perren SM. Importance of the friction between plate and bone in the anchoring of plates for osteosynthesis. Determination of the coefficient of metal-bone friction in animal in vivo. Helv Chir Acta. 1979;46:183-7.

22. Goldstein SA, Wilson DL, Sonstegard DA, Matthews LS. The mechanical properties of human tibial trabecular bone as a function of metaphyseal location. J Biomech. 1983;16:965-9.

23. Townsend PR, Rose RM, Radin EL. Buckling studies of single human trabeculae. J Biomech. 1975;8:199-201.

24. Meakin JR, Shrive NG, Frank CB, Hart DA. Finite element analysis of the meniscus: the influence of geometry and material properties on its behaviour. Knee. 2003;10:33-41.

25. Kumar D, Manal KT, Rudolph KS. Knee joint loading during gait in healthy controls and individuals with knee osteoarthritis. Osteoarthr Cartil. 2013;21: 298-305.

26. Luo CA, Lin SC, Hwa SY, Chen CM, Tseng CS. Biomechanical effects of plate area and locking screw on medial open tibial osteotomy. Comput Methods Biomech Biomed Engin. 2015;18:1263-71.

27. Diffo Kaze A, Maas S, Waldmann D, Zilian A, Dueck K, Pape D. Biomechanical properties of five different currently used implants for openwedge high tibial osteotomy. J Exp Orthop. 2015;2:14.

28. Han SB, Bae JH, Lee SJ, Jung TG, Kim KH, Kwon JH, et al. Biomechanical properties of a new anatomical locking metal block plate for opening wedge high tibial osteotomy: uniplane osteotomy. Knee Surg Relat Res. 2014;26:155-61.

29. Bottlang M, Doornink J, Fitzpatrick DC, Madey SM. Far cortical locking can reduce stiffness of locked plating constructs while retaining construct strength. J Bone Joint Surg Am. 2009;91:1985-94.

\section{Publisher's Note}

Springer Nature remains neutral with regard to jurisdictional claims in published maps and institutional affiliations.

Ready to submit your research? Choose BMC and benefit from:

- fast, convenient online submission

- thorough peer review by experienced researchers in your field

- rapid publication on acceptance

- support for research data, including large and complex data types

- gold Open Access which fosters wider collaboration and increased citations

- maximum visibility for your research: over $100 \mathrm{M}$ website views per year

At $\mathrm{BMC}$, research is always in progress.

Learn more biomedcentral.com/submissions 